e dit questa, per essere, in rirtu delle $(\bar{\sigma} a)\left(\mathcal{Z}^{\prime}\right)$ e $\left(4^{\prime}\right)$ :

$$
\begin{gathered}
\varepsilon\left(\frac{\partial \mathbf{F}}{\partial p^{\prime}}+\frac{\partial \mathbf{F}^{\prime \prime}}{\partial p^{\prime \prime}}\right)=-4 \pi \varepsilon \frac{\partial \mathbf{e}}{\partial t}=\frac{4 \pi}{\mu}\left(\mathbf{u}^{\prime} \alpha^{\prime}+\nabla \beta^{\prime}+\mathbf{w}^{\prime} \gamma^{\prime}\right. \\
\left.+\mathbf{u}^{\prime \prime} \alpha^{\prime \prime}+\mathbf{v}^{\prime \prime} \beta^{\prime \prime}+\mathbf{w}^{\prime \prime} \gamma^{\prime \prime}\right)=4 \pi\left(u^{\prime} \alpha^{\prime}+v^{\prime} \beta^{\prime}+v^{\prime} \gamma^{\prime}+u^{\prime \prime} x^{\prime \prime}+v^{\prime \prime} \beta^{\prime \prime}+w^{\prime \prime} \gamma^{\prime \prime}\right),
\end{gathered}
$$

si ricava la (11), come doverasi dimostrare.

\title{
SUL FENOMENO DI HALL NEI LIQUUDI.
}

Nota del Prof. HORTUNATO FLORIO.

In una sua Nota ${ }^{1}$ ) Bagard espose delle esperienze dalle quali apparirebbe che, contrariamente ai risultati avuti dal Prof. Róiti ${ }^{2}$ ), il tenomeno di Hall si produce nei liquidi. A me parve che colla disposizione alottata lal Bagard in tali esperienze si fossero arute diverse cause d'errore, per cui ne ideai un' altra ${ }^{3}$ ) che ne fosse esente e con essa ebbi risultati negativi. Bagard riprese in seguito le sue ricerche ") senza cambiare sostanzialmente lid disposizione primitiva, ottenne dei pisultati concordanti con quelli già aruti, e, comparsa la mia Nota, volle criticarla ${ }^{5}$ ). Adesso non credo inutile dire quel che segue.

Nella sua $2^{3}$ Nota Bagard considera un puuto $M$ d'una lamina liquida studiata, la retta $\mathrm{MX}$ che, passando per $\mathrm{M}$, è parallela alla direzione del flusso elettrico prima di eccitare il campo magnetico, e la retta MY perpendicolare ad MLX e alla direzione del flusso magnetico, il quale attraversa normalmente la I mina. L'azione del campo magnetico secondo l' Autore avrebbe per effetto di orientare la forza elettrica secondo una direzione MF, ene farebbe un angolo D con la MX. Egli chiama con $\mathrm{X}$ e $\mathrm{Y}$ le componenti di tale forza nelle direzioni $\mathrm{MX}$ ed $\mathrm{MY}$, e determina $\mathrm{X}$ misurando la differenza di potenziale

1) Comptes Rendus, 13 lienuaio 1896

2) Atti della R. Acc. doi Lincei, 3. serie, t. 12, pag. 397; 1882.

3) Nuovo Cimento, Agosto 1896.

4) Journal de Physique, Novembre 1896.

5) Comptes Rendus, 29 Dicembre 1896; Nuovo Cimento, Dieembre 1896. 
tra 2 scandagli applicati allit lamina in due punti lungo MX e dividendola per la distanza che separa questi; in modo analogo, adoperando 2 alti scandagli, determina Y. Bagard aggiunge che, essendo impossibile il collocare esattamente gli scandagli nelle direzioni suddette, chiama con $x^{\prime} l^{\prime}$ angolo che la $\mathrm{MX}$ fa colla retta che unisce i punti in cui sono applicati $\mathrm{i}$ due primi scandagli, e con $x$ quello che la MY fa colla retta che unisce $\mathrm{i}$ punti in cui sono applicati gli altri due scandagli.

A me pare evidente che se nelle esperienze alle quali l'Autore si riferisce non si fossero avute cause d'errore, gli angoli $x$ ed $\alpha^{\circ}$ per un dato apparecchio e per una data soluzione avrebbero dovuto rimanere costant; invece egli trova che a gli varia fino di $6^{\prime} 50^{\prime \prime}$ (V. $2^{a}$ Nota, pag. 507, specchietto corrispondente ad $n=2$ ) in una stessa esperienza, durante la quale quindi il flusso elettrico nella lamina studiata arrebbe variato continuamente di direzione indipendentemente dal lenomeno di Hall: egli non ha preso nessun provredimento per ovriare a ciò.

Inoltre nel ricercare le espressioni di a e di I, l'Autore combina le 4 equazioni:

$\mathrm{X}_{1}=\mathrm{X}\left(1+\alpha^{\prime} \mathrm{D}\right), \quad \mathrm{Y}_{1}=\mathrm{X}(\mathrm{D}-\alpha), \quad \mathrm{X}_{2}=\mathrm{X}\left(1-x^{\prime} \mathrm{D}\right), \quad \mathrm{Y}_{2}=-\mathrm{X}(\mathrm{D}+\alpha)$

fove $\mathrm{X}_{1}$ e $\mathrm{Y}_{1}$ sarebbero $\mathrm{i}$ valori delle componenti della forza clettrica in $\mathrm{V}$ secondo le rette che uniscono i punti di applicazione degli seandagli di ciascuna coppla quando l' elettrocalamita $\dot{e}$ eccitata in un certo senso, eX e Y quelli della stessa forta secondo $M X$ e WY ed $x_{z}$ ed $Y_{z}$ i ralori analoghi ad $X_{1}$ e $Y_{1}$ e colrispondenti al caso in cui dopo arer determinato $x_{1}$ e $Y_{1}$ soltanto s' inverta la direzione del campo magneticos e ció nell' ipotesi che $x$ e $x^{\prime}$ nell' intervallo (10 minuti) che faceva decorrere fra l'istante in cui facera le leflure per $\mathrm{x}_{1}$ e $\mathrm{r}_{1}$ e quello in eui ficeva le letture per $\mathrm{X}_{2}$ e $\mathrm{Y}$, lossero rimasti inalterati, sicchi le expressioni trovate da lui per $x$ e fer 1', colle quali ha misumato l'eflettr Hall, non credo possano applicari alle sue coperienze.

I lisultati di Batrard dietro quanto ho deto credo che restino abbastanza scoksi, e arlesw mi propongo di exporre lo

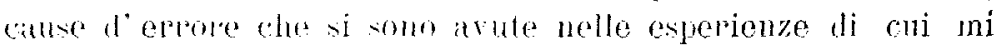


sono occupato, a precehie delle quali mi pare si possa atrihuile la valiabiliti di $x$ e di $x$.

Prima d'incominciare le slie oxervazioni Bagald larciava fassate attraverso alla lamina lifuida la corrente grer un'ora: questo tempo ela sufficiente perchè la soluzione che studiava fosse divenuta sensibilmente eterogenea per il noto fenomeno hel trasporto degli ioni e fosse continuata ad esserlo magyiormente durante lo esperienze. Talc eterogeneita, che muta col tompo, l' assottigliarsi di un elettrodo e l'ingrossirsi dell' altro, l'azione delle scosse ece. potevano durante i 10 che Bagard ticeva deconrere dall istante in cui ficera le misure per $\mathrm{X}_{1}$ e $\mathrm{I}_{1}$ e quello in cui liacera le misure per $\mathrm{X}_{2}$ e $\mathrm{Y}_{2}$ far rapiare le differenze di potenziale arrlj scandagli di ciascuma coppia indipendentemente ral fenomeno di Hall.

Noteri poi che nel ripetere le esperienze di Bagard arevo osservato la formazione di filamenti metallici e di depositi metallici al disotto degli elettrodj mobili, variabili di forma e di dimensioni, sensibili alle axioni magneticho el elettrodinamiche ed alle scosse: Bagard stescio dice ${ }^{1}$ ) di aver notato la tor'mazione di piccoli aghi sulla superficie degli eletrodi di zinco quando la corrente ara una densita notevole.

Il deposito metallico sugli elettrodi essendo arvenuto in regioni ove il campo magnetico era molto intenso, potrebhe darsi che le particelle metalliche all atto di depositalsi avessero sentita un' azione orientatrice che per diverse rayioni arrebbe potuto influire sui risultati finali. Il liquido nelle vaschette adiacenti alla lamina, nelle quali non aveva un' altezza superiore di molto allo spessore della lamina, da Bagard era fat to terminare con superficie libera e veniva a trovarsi in campi magnetici intensi: è noto che il l’of. Roiti avea già trovato ${ }^{2}$ ) che cio costituisce una buona causa d'errore. Le lamine metalliche, che funzionarano da elethodi, poste o sottratte insieme alla lamina liquida da campi magnetici intensi

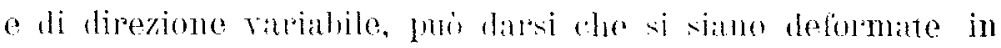

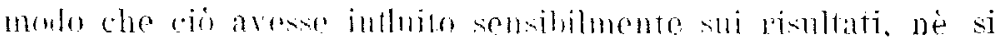

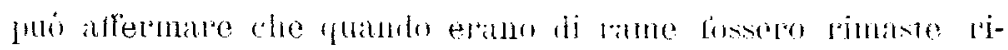

1) Nuovo Cimento, Hicenbre 1s96.

2) $1.1 . \mathrm{os}$ 
gide, griachè all' infuori delle azioni elettrodinamiche è noto quanto sia difficile trovare del rame insensibile alle azioni magnetiche.

Quello che ho arauti detto credo che sia sufficiente per spiegare eio che nella sua la Nota Bayard chiama effetto progressivo dell' elotrocalamita e che diflicilmente mi pare che si possa spiegare, come tia Bagard, invocando l' effetto Joule, giacche ratiando questo pressoche mitormemente, non si capisce perchè per la sua azione, stabilendo la corrente nell' olottrocalamita, la diflerenzia di potenziale debba in ogni esperienza variare rapidamente durante il $1^{\circ}$ minuto, poi piu lentamente durante i minuti seguenti e fissarsi generalmente dopo il $3^{\circ}$ o $4^{0}$ minuto.

A principio della sua 2 Nota Bagard dice che per eliminare le perturbazioni che provengono dal riscaldamento Joule egli é stato conlotto a prendere degli spessori sempre piu grandi per la lamina liquida : aumentando cosi la sezione della lamina, aggiunge, si puó infatti con una f. e. m. data rendere la densità della corrento e per conseguenza l' effetto Joule suflicientemente debole ecc.

Io osservo che se si suppone di mantenere costante la diflerenza di potenziale tra gli strati che separano la lamina dal resto della soluzione mentre si fa variare lo suessore della lamina, è chiaro che la densita della corrente non mutera e con essa l'effetto Joule. Non mi jare dunque che coll'assegnare spessori esagerati alle lamine, si possano sopprimere le suddette perturbazioni.

Mi sembrano un po' strani alcuni dei risultati ottenuti da Bagard : cosi p. e. esaminando lo specchiettino corrispomentes al $n=2$ (2) Notal pag. 507) si trovia cho nel passaggerio dalla sit alla ja esperienza, solo perche il campo magnetico passava da 707 a 962 uniti (G. (4. S.) l' effetto Hall D andava da $20^{\prime \prime}$ a $0^{\prime \prime}$, mentre per' la sa delle conchusoni che sono in fine di detia nota in tale passaggio ci doverbbe onsere un accrencimonto di 1 .

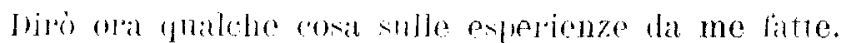

Rimando il lettome allit mia Nota avanti citata pere la disposizione ch io nelle mie ricerche adottai: dirò solo che gli 
elettrodi nel caso che esaminato delle soluzioni di solfato di zinco erano formati da analgama liquida di zinco od elano posti molto lontano dalla lamina e sottratti a qualsiasi sensibile arione magnetica: in molo analogo procedevo quando esaminavo altre soluzioni.

Mi sj rimprovera che tale disposizione sia difettosa perchè io tacevo entrare ed uscire la corrente da due vertici opposti della lamina, che era rettangolare, mentre agli altri due erano applicati gli scandagli, ma credo che un til modo di sperimentare non differisca sostanzialmente da quello che è stato adottato per lo studio dello stesso fenomeno nelle lamine meialliche, le quali spesso sono state latte di forma circolare e nelle quali si $\dot{e}$ latta entrare ed uscire la corrente in tratticini posti in vicinanza delle estremita di un loro diametro, mentre gli scandagli sono stati applicati in corrispondenza delle estremiti dell'altro diametro perpendicolare al precedente.

Relativamente all" ultima disposizione adottata da Bagard ${ }^{1}$ ) nella quale l'Autore adopera degli elettrodi di amalgama di zinco liquida posti fuori del campo magnetico, noteró che il modo di sperimentare essendo lo stesso, come egli asserisce, di quello prima tenuto, parecthe delle osservazioni avanti fatte si possono ad essa riferire, ed aggiungero che con tale disposizione in conchusione non veniva a studiare che una lamina liquida, che nella parte centrale area uno spessore metà che nelle parti laterali, e che in queste la soluzione era sottoposta ad un'azione magnetica molto meno intensa che in quella e che inoltre vi terminara con superficie libera. con tale disposizione inoltre pigliavano parte attiva soltanto delle porzioni abbastanza limitate degli elettrodi vicine alla lamina, il che oltre a rendere inulile il porredimento preso di asseguare a tali elettrodi la lumghezza di jom. avtri fatto si the al disopra di essi la soluzione fosse divenuta eterogenea lungo piani ofizzontali, e i moti di agitazione, faroriti dalla presenza dell amalgama liquida, araanno probabilmente fatto s'ntire in modo sensibile la loro influenza.

1) Nuovo Cimtento, Jicemlire 1sy6; Comptes Rendus, 20 Dicembre 1nafi. 
Dietro quanto ho detio mi pare di poter concludere che dalle riceloche di Barard nom si pro con sicurezza dedurte che il fenomeno di Hall xi produca nei liquidi: i risultati da me otenuti concoldantemente a ruelli dal Prol. Roitj lurono negatiri, e, dolente che le mie condizioni non mi abbiano permeson di jortare un nuovo contributo sierimentale alla risoluzione della quistione, non protrei adessoche ritenere questa come non risolta.

R. Istituto tecnico di Cagliari, Marzo 1896.

\section{DELLE AZIONI DELL' ELETTRICITA SULLA VIRTÙ SCARIGATRICE INDOTTA NELL' ARIA DAI RAGGI $\mathbf{X}$.}

Nota di EMILIO VILLARI ${ }^{1}$ ).

E noto che $\mathrm{i}$ gas attraversati dai magi $\mathrm{x}$ od $\mathrm{x}$ ati acruistano li proprieti di scarjearo i comblutori eletrizati. Io ho

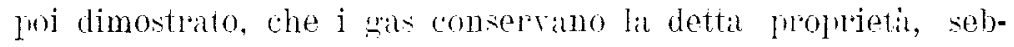
bene dimjunita, anche dopo arep percosso dej tuhi di retro o di piombo di lo o jui metri di lunghezza; ma lil perdono completamente pasimelo jel un ozomatore in ativiti, o di recente stato ativato. Questa propricta noutralizzante dell ozomatole deruta allo cariche residue, risvegliateri dall efhuvio elettrico, le quali con lentezza si disperdono. Ho puscia seruitato ruesti stuli, e qui expon i joincipali risultati ai quali sono perremuto.

L'allatechio che misi insieme fu oggetto di speciali cure, fur aminare le diverse influnze perturbatrici. Esso es schematicamente indicato in planta dalla ligura 1.

Cna cassa cubica di zinco $77 . X X$, di 1 metoo di lato is pu-

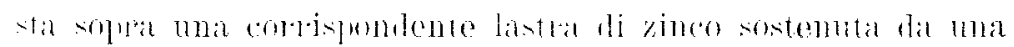

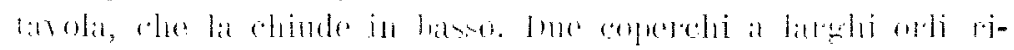

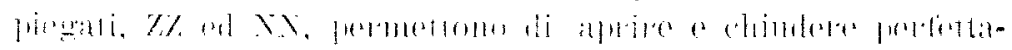

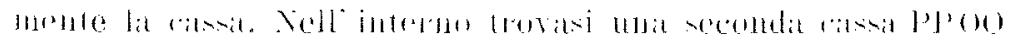

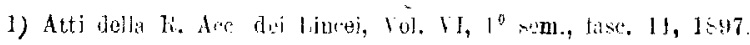

Serie IV, Fol. Irl. 